\title{
Nutritional Diversity of Rhizobiaceae Revealed by Auxanography
}

\author{
By DONNA PARKE* AND L. NICHOLAS ORNSTON \\ Biology Department, Box 6666, Yale University, New Haven, CT 06511, USA
}

(Received 23 November 1983; revised 26 March 1984)

\begin{abstract}
Many aromatic compounds are toxic when supplied at concentrations employed in most growth media. This effect was demonstrated when rhizobia and agrobacteria were grown in auxanographic plates in which cells were seeded in agar and exposed to a gentle gradient of substrate concentration. An auxanographic nutritional survey with representative strains revealed that Rhizobium japonicum and cowpea Rhizobium sp. could utilize a relatively large proportion of the aromatic and hydroaromatic compounds tested; Rhizobium leguminosarum, Rhizobium trifolii and Agrobacterium species displayed intermediate nutritional versatility; Rhizobium meliloti was relatively fastidious. The hydroaromatics quinate and shikimate were not toxic. Most of the strains examined grew at the expense of one or both of these substrates. Quinate was metabolized via protocatechuate and 3-oxoadipate. All of the strains examined were able to grow at the expense of protocatechuate and therefore must contain six structural genes for the enzymes required to convert this aromatic compound to common intermediary metabolites. Conservation of pathways for aromatic catabolism against a background of wide evolutionary divergence among the Rhizobiaceae suggests that pressures for selection of the traits were exerted throughout the evolutionary history of the organisms. A probable selective pressure is competition for nutrients in the soil. In addition, the ability of agrobacteria and rhizobia to respond to aromatic compounds may have selective value in bacterial-plant interactions.
\end{abstract}

\section{INTRODUCTION}

Nutritional surveys contributed to the classification of rhizobia (Graham, 1964a; Graham \& Parker, 1964; Moffett \& Colwell, 1968) and agrobacteria (Keane et al., 1970; White, 1972; Kersters et al., 1973). Patterns of carbohydrate utilization suggested that relatively fast growing rhizobia and agrobacteria are nutritionally diverse whereas slow growing rhizobia appear to be nutritionally fastidious. It is clear from the work of Parker et al. (1977) and Glenn \& Dilworth (1981) that some rhizobial strains grow at the expense of aromatic compounds, yet most aromatic growth substrates have been overlooked in previous nutritional surveys. This investigation was undertaken to see if genes for the catabolism of aromatic compounds are distributed widely among members of the Rhizobiaceae.

Early in our work it became apparent that most aromatic compounds are toxic to members of the Rhizobiaceae. In order to explore the full genetic potential of strains, the compounds were supplied in low concentration by auxanography in a defined minimal medium. A similar method was employed by Graham (1964b) to observe growth supplementation of rhizobia and agrobacteria by carbohydrates and Krebs cycle intermediates in an undefined medium. The work reported here, the first systematic survey of the Rhizobiaceae employing aromatic, hydroaromatic and related compounds, demonstrates substantial catabolic potential within the biological group and indicates that the slow growing strains may be more nutritionally versatile than previously recognized.

Abbreviations: MM, defined minimal medium; YM, yeast extract/mannitol medium. 
Table 1. Bacterial strains

Species

Fast growing strains

Rhizobium meliloti

R. meliloti

Rhizobium leguminosarum

R. leguminosarum

Rhizobium trifolii

R. trifolii

R. trifolii

R. trifolii

Rhizobium japonicum

Agrobacterium tumefaciens

Agrobacterium rhizogenes

Slow growing strains

Rhizobium japonicum

$R$. japonicum

R. japonicum

Cowpea Rhizobium sp.

Cowpea Rhizobium sp.

Cowpea Rhizobium sp.
Strain

102F28

FA1021

2368

2376

2066

$162 \mathrm{~S} 33$

BP100

HM100

192

B6

A4

$61 \mathrm{~A} 76$

110

123

3179

3241

$32 \mathrm{HI}$
Source

J. C. Burton \& R. S. Smith, The Nitragen Co. Inc., Milwaukee, Wisconsin 53209, USA

F. M. Ausubel, Harvard University

H. H. Keyser, USDA, Nitrogen Fixation Lab., Beltsville, MD 20705, USA

J. C. Burton \& R. S. Smith, The Nitragen Co. Inc. Trifolium repens $\mathrm{L}$. nodules; Hamden, $\mathrm{Ct}$., USA Trifolium pratense $\mathrm{L}$. nodules; Hamden, Ct., USA H. H. Keyser, USDA, Nitrogen Fixation Lab.

S. R. Long, Stanford University

D. Tepfer, CNRA, Versailles, France \}

J. C. Burton \& R. S. Smith, The Nitragen Co. Inc.

H. H. Keyser, USDA, Nitrogen Fixation Lab.

R. S. Smith, The Nitragen Co. Inc.

\section{METHODS}

Media and growth conditions. Bacterial strains used in this study are listed in Table 1. Fast growing strains are defined as those which have generation times of 2 to $4 \mathrm{~h}$ on yeast extract/mannitol medium, whereas slow growing strains are those with generation times of $6 \mathrm{~h}$ or more (Vincent, 1977). The organisms were maintained on plates containing yeast extract/mannitol medium (YM; Wacek \& Brill, 1976). Colonies were picked from YM plates and placed in a defined liquid minimal medium (MM; Ornston \& Stanier, 1966) containing Hutner's 'Metals 44' (Cohen-Bazire et al., 1957) and biotin at $0.5 \mu \mathrm{g} \mathrm{ml}^{-1}$; the medium was modified in that it contained $0.0125 \mathrm{M}$ each of $\mathrm{KH}_{2} \mathrm{PO}_{4}$ and $\mathrm{Na}_{2} \mathrm{HPO}_{4}$. The minimal medium for strains of $R$. leguminosarum and $R$. trifolii contained additional supplements of thiamin. $\mathrm{HCl}$ at $1 \mu \mathrm{g} \mathrm{ml}^{-1}$ and calcium pantothenate at $2 \mu \mathrm{g} \mathrm{ml}^{-1}$. Carbon sources were prepared as $0.5 \mathrm{M}$ or $1.0 \mathrm{M}$ concentrated solutions, neutralized with $\mathrm{NaOH}$, and filter sterilized. Catechol and sodium benzoate were obtained from MCB Manufacturing Chemists Inc., Cincinnati, Ohio 45212, USA. cis, cisMuconate was synthesized chemically (Riegel \& Lilienfeld, 1945), and all other carbon sources were purchased from Sigma. Liquid cultures were incubated at $30^{\circ} \mathrm{C}$ on a gyrotory New Brunswick Environmental shaker at 240 r.p.m. Growth was measured by monitoring optical density at $600 \mathrm{~nm}$.

Auxanography (Lederberg, 1946) was used to screen strains for their ability to utilize aromatic compounds as a source of carbon and energy. Cells were grown in $5 \mathrm{ml}$ growth tubes to early stationary phase, corresponding to an optical density of 0.7 at $600 \mathrm{~nm}$, using $10 \mathrm{~mm}$-succinate as a growth substrate. Rhizobium japonicum strain 192, $R$. leguminosarum and $R$. trifolii were exceptions in that they were grown on media containing $10 \mathrm{~mm}$-glucose, arabinose or mannitol. Cells were rinsed with an equal volume of MM lacking carbon source. Resuspended cells were then mixed with MM containing $1.5 \%(\mathrm{w} / \mathrm{v})$ purified Noble agar (Difco) at a temperature below $50{ }^{\circ} \mathrm{C}$, and $20 \mathrm{ml}$ of the media containing the cells was poured into each $15 \times 100 \mathrm{~mm}$ Petri dish. Each Petri dish received approximately $2 \times 10^{9}$ cells. The plates were left overnight to solidify before carbon sources were added. Up to three different carbon sources were spotted onto the edge of a plate using 15 to $30 \mu \mathrm{mol}$ of each substrate; each carbon source was tested on at least three plates. Plates were incubated at $30^{\circ} \mathrm{C}$ for up to $4 \mathrm{~d}$. All of the above operations were carried out under aseptic conditions.

Contamination was routinely checked by microscopic examination of cultures and by streaking cells onto a YM plate containing $0.0025 \%$ Congo red. Most of the strains used in this study were distinct in morphology and growth rates. In addition, strains were tested for their ability to nodulate appropriate host plants.

Nodulation tests. Rhizobial strains were examined for symbiotic properties by inoculation onto seedlings. Seeds were surface sterilized by brief immersion in $70 \%(\mathrm{v} / \mathrm{v})$ ethanol, rinsing with sterile distilled water, treatment with $50 \%(\mathrm{v} / \mathrm{v})$ Chlorox for 10 or $15 \mathrm{~min}$, and rinsing six more times with sterile distilled water. Seeds of 'Siratro' and vetch were pretreated with concentrated $\mathrm{H}_{2} \mathrm{SO}_{4}$ for $10 \mathrm{~min}$. They were rinsed with sterile distilled water nine times prior to the Chlorox treatment. Seeds were germinated on sterile agar slants in tubes supplied with Jensen's medium (Vincent, 1970). Plants were grown for 4 to 6 weeks in a growth chamber at $24^{\circ} \mathrm{C}, 18000 \mathrm{~lx}$, and with a $16 \mathrm{~h}$ photoperiod. Rhizobia were grown in liquid YM medium, rinsed, and resuspended in distilled water. Week old plants were inoculated with approximately $10^{7}$ bacteria in $0.5 \mathrm{ml}$. 
The host plant nodulated by all of the Rhizobium japonicum and cowpea Rhizobium sp. strains was 'Siratro' (Macroptilium atropurpureum, PI no. 316463). Vetch, Vicia sativa cv. 'Woodford', formed nodules when inoculated with the Rhizobium leguminosarum strains, and Trifolium pratense var. 'Pennscott' was nodulated by all strains of Rhizobium trifolii except strain 162S33. Medicago sativa var. 'Iroquois' was the host of Rhizobium meliloti strains. 'Siratro' and vetch seeds were kindly provided by Gill Lovell of the USDA Regional Plant Introduction Station, Experiment, Ga. 30212, USA. Other seeds were obtained from Agway Inc.

\section{RESULTS}

Genetic potential revealed by auxanography. Auxanography revealed the genetic potential of strains to grow with compounds that were toxic at high concentrations. Anthranilate, benzoate, cinnamate, catechol, $p$-coumarate, gallate, gentisate, mandelate, $p$-hydroxybenzoate, syringate and vanillate supported growth at low substrate concentration, revealed by an arc of growth away from the origin of substrate application to the plate (Fig. 1). The toxicity of the compounds was reflected in the distance of the arc from the origin, and it varied from strain to strain. In some cases growth was limited to a narrow band and, in others, a broad band (Fig. 1).

Auxanography also distinguished between mutation or variation in a minority of cells and the slow growth of an entire population, a distinction which might have been overlooked in liquid cultures. For example, all of the $R$. trifolii, $R$. leguminosarum and Agrobacterium tumefaciens strains formed mutant or variant colonies readily when exposed to shikimate (Table 2), and thus the wild type cells possessed all the genes required for efficient utilization of this substrate.

Protocatechuate, a universal growth substrate for rhizobia. Protocatechuate supported the growth of all the strains included in this survey. The compound is relatively nontoxic to many strains, but its chemical instability makes it undesirable as a growth substrate for metabolic studies. On the other hand, quinate or shikimate, stable nontoxic growth substrates which are utilized via protocatechuate (Fig. 2), supported growth of almost all the examined strains. A representative of each species that utilized quinate was shown to convert it to 3-oxoadipate, determined as material giving a positive Rothera reaction (Evans, 1947; Stanier et al., 1966). This evidence indicated that quinate and protocatechuate were metabolized via the pathway outlined in Fig. 2.

Growth substrates that distinguish between fast growing and slow growing strains. The survey revealed that the slow growing strains examined grew at the expense of adipate whereas fast growing strains did not. Adipate, a nonmetabolizable analogue of the inducer 3-oxoadipate in some Pseudomonas species (Ornston \& Parke, 1976), was nontoxic and thus might be used to facilitate isolation of slow growing rhizobia from the soil without interference from fast growing rhizobia or agrobacteria.
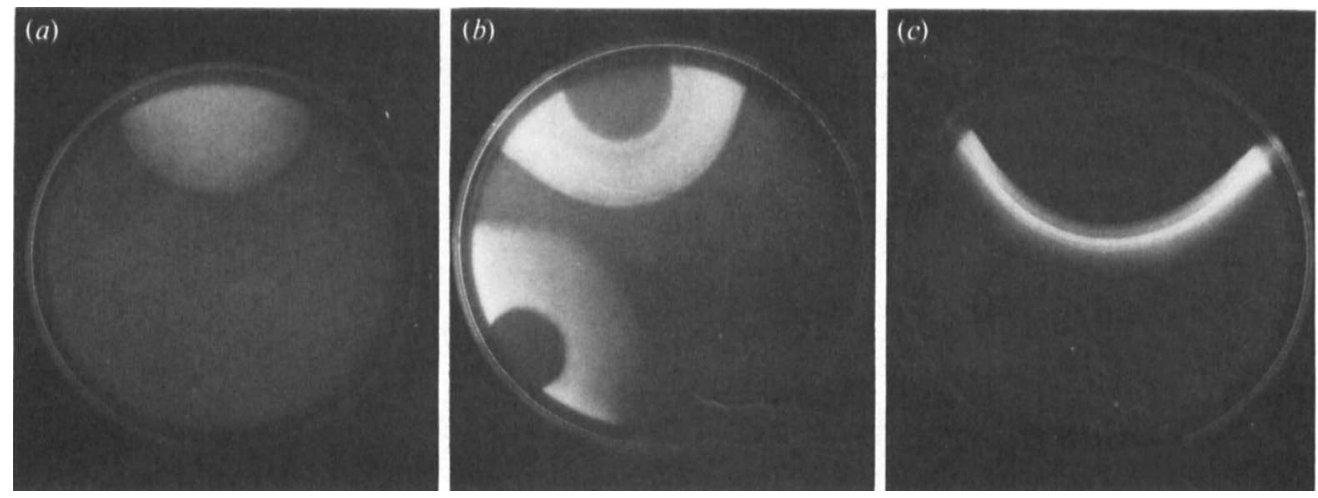

Fig. 1. Growth patterns on auxanographic plates. (a) Growth of $A$. tumefaciens with quinate, a nontoxic substrate. (b) Arcs observed after growth of $R$. leguminosarum strain 2376 with moderately toxic substrates benzoate and $p$-hydroxybenzoate. $(c)$ Narrow arc observed after growth of $R$. meliloti strain $102 F 28$ with the extremely toxic substrate anthranilate. 


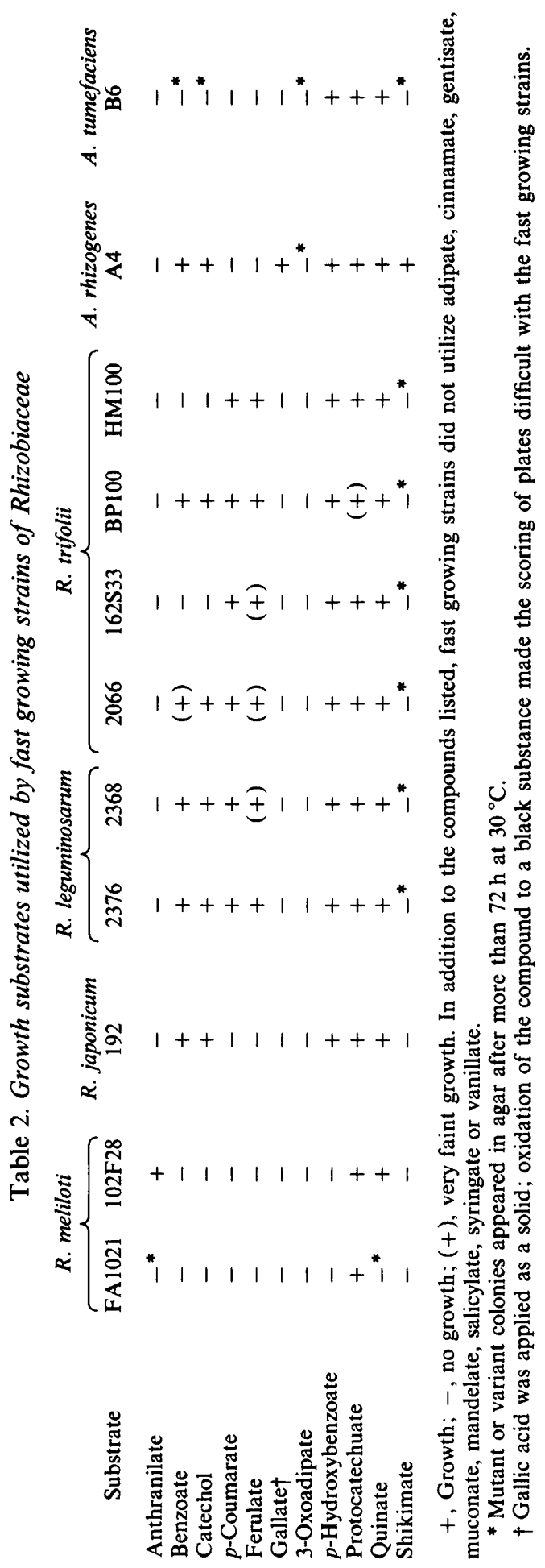




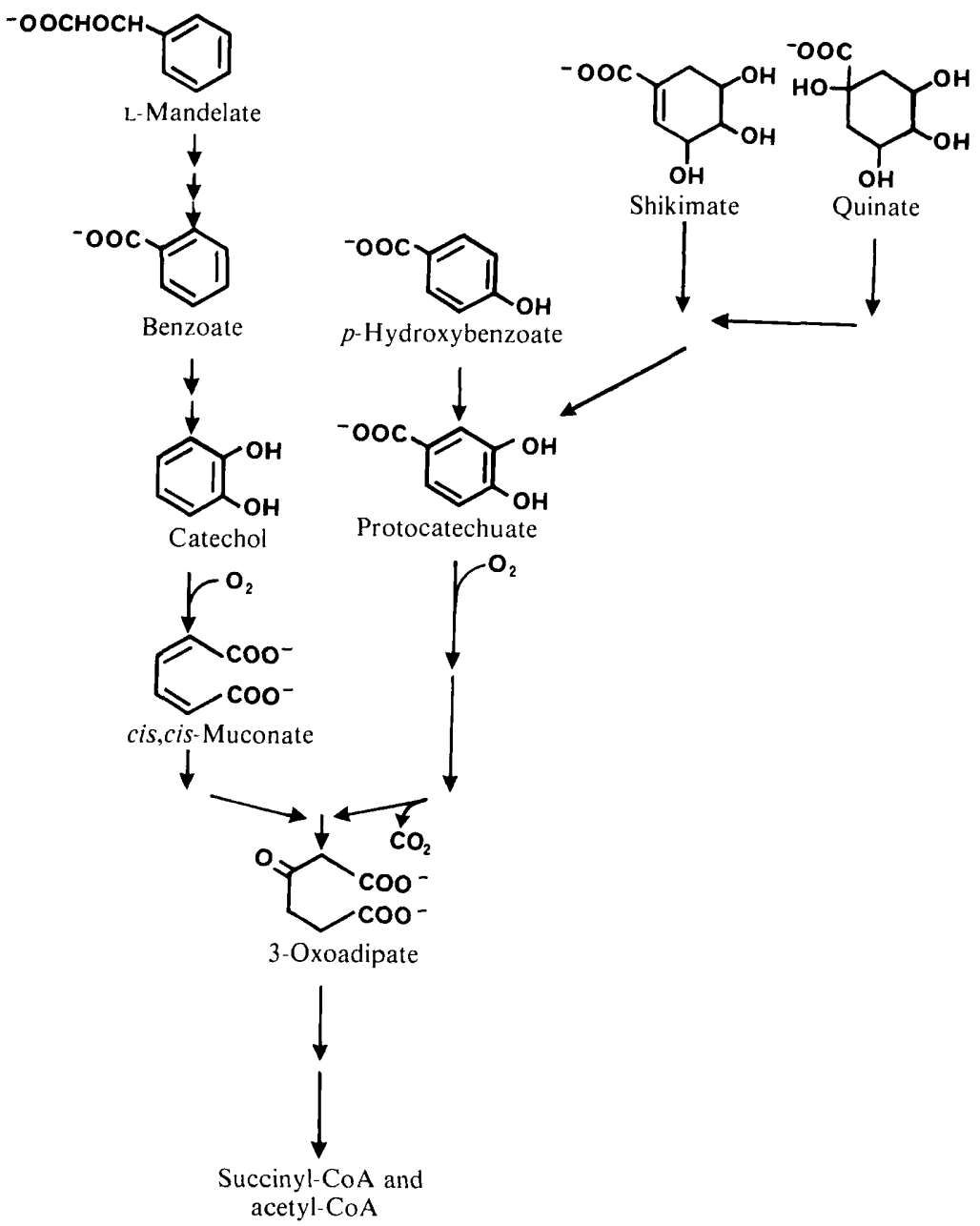

Fig. 2. Pathways for the catabolism of aromatic and hydroaromatic compounds via 3-oxoadipate in prokaryotes. Each arrow represents a specific enzymic step. As shown in this paper, quinate is degraded to 3-oxoadipate by agrobacteria and rhizobia. Pathways used by the organisms for catabolism of most of the other compounds included in this study remain to be determined.

All of the slow growing strains except possibly Rhizobium sp. strain $32 \mathrm{H} 1$ grew at the expense of vanillate whereas the fast growing strains did not. It should be noted that other workers have reported a fast growing rhizobial strain which was able to utilize a wide range of aromatics including vanillate (Glenn \& Dilworth, 1981).

Growth patterns of fast growing strains. The fast growers could be distinguished among themselves by their responses to ten aromatic and hydroaromatic compounds (Table 2). The two $R$. meliloti strains were most fastidious with respect to these compounds, demonstrating the genetic potential to utilize only three of them (Table 2). Protocatechuate was the sole tested compound that supported the growth of $R$. meliloti strain FA1021, but the organism readily acquired the ability to grow with either anthranilate or quinate. Thus the nutritional potential of this strain was identical to that of the independent $R$. meliloti isolate, strain 102F28 (Table 2).

Two $R$. leguminosarum strains and four $R$. trifolii strains shared almost the same catabolic pattern, utilizing seven of the ten compounds. The only difference between the two species was failure of two of the $R$. trifolii strains to share a reproducibly positive growth response with benzoate (Table 2). R. japonicum 192, a fast growing isolate from China (Keyser et al., 1982) was 
Table 3. Growth substrates utilized by slow growing rhizobial strains

\begin{tabular}{|c|c|c|c|c|c|c|}
\hline Substrate & $\begin{array}{l}\text { R. japonicum } \\
61 \mathrm{~A} 76\end{array}$ & $\begin{array}{c}R . \text { japonicum } \\
110\end{array}$ & $\begin{array}{c}\text { R. japonicum } \\
123\end{array}$ & $\begin{array}{c}\text { Rhizobium } \\
\text { sp. } \\
3179\end{array}$ & $\begin{array}{c}\text { Rhizobium } \\
\text { sp. } \\
3241\end{array}$ & $\begin{array}{c}\text { Rhizobium } \\
\text { sp. } \\
32 \mathrm{H} 1\end{array}$ \\
\hline Adipate & + & + & + & + & + & + \\
\hline Anthranilate & + & + & $(+)$ & $(+)$ & $(+)$ & $(+)$ \\
\hline Benzoate & + & + & $(+)$ & - & - & $(+)$ \\
\hline Cinnamate & + & $(+)$ & $(+)$ & - & - & $(+)$ \\
\hline Catechol & + & - & - & - & - & - \\
\hline$p$-Coumarate & + & + & + & + & + & + \\
\hline Ferulate & + & + & + & + & + & $(+)$ \\
\hline Gallate* & + & + & + & + & + & + \\
\hline Gentisate & + & + & + & + & + & + \\
\hline 3-Oxoadipate & + & + & + & + & + & + \\
\hline Mandelate & + & $(+)$ & $(+)$ & - & $(+)$ & $(+)$ \\
\hline cis,cis-Muconate & + & - & - & - & - & - \\
\hline$p$-Hydroxybenzoate & + & + & $(+)$ & + & + & $(+)$ \\
\hline Protocatechuate & + & + & + & + & + & + \\
\hline Quinate & - & + & + & + & + & + \\
\hline Shikimate & - & + & + & + & + & + \\
\hline Syringate & - & + & + & $(+)$ & - & - \\
\hline Vanillate & + & + & + & + & + & $(+)$ \\
\hline
\end{tabular}

+ , Growth; -, no growth; (+), very faint growth. Salicylate did not serve as a growth substrate for any of the above strains.

* Gallic acid was applied as a solid.

similar to the $R$. leguminosarum strains except that it did not mutate to growth at the expense of shikimate and did not grow with $p$-coumarate or ferulate (Table 2).

To test whether strains had lost the ability to utilize any of the tested compounds during longterm storage, two $R$. trifolii strains, BP100 and HM100, were isolated from nodules of native white and red clover plants, respectively, and subjected to auxanography after being subcultured once on YM. Their growth patterns were similar to those of $R$. leguminosarum and $R$. trifolii strains from culture collections.

Representatives of the genus Agrobacterium have been classified into two major biotypes on the basis of biochemical tests and their differential ability to utilize several growth substrates (Elkan, 1981). A representative of biotype 1, A. tumefaciens B6, and one of biotype 2, A. rhizogenes A4, were included in this survey. The two Agrobacterium strains were distinguishable from the leguminosarum-trifolii group by their failure to utilize $p$-coumarate and ferulate and by the ability of the $\boldsymbol{A}$. rhizogenes strain to catabolize gallate. With respect to other compounds tested, the two agrobacteria appeared to be more closely related to the leguminosarum-trifolii strains than they were to the R. meliloti strains (Table 2). This contrasts with the conclusions of White (1972) and Graham (1964a) who found that, on the basis of numerous criteria, both Agrobacterium biotypes were more closely related to $R$. meliloti than to $R$. leguminosarum.

Growth patterns of slow growing strains. Whereas fast growing strains demonstrated the ability to utilize from one to seven of the proffered substrates, slow growing strains showed a relatively full growth yield at the expense of from eight to fifteen of the compounds (Table 3). The three $R$. japonicum strains included in the survey fall into different DNA homology groups within this species (Hollis et al., 1981). The cowpea Rhizobium sp. strain 3179, also known as 3G4b4, may bear a subspecies relationship to $R$. japonicum strains 110 and 123 , whereas Rhizobium sp. strain $32 \mathrm{H} 1$ falls outside this group (Hollis et al., 1981). Thus, across a broad spectrum of DNA homology groups, slow growing rhizobia demonstrated the capacity to utilize aromatic compounds and showed different patterns of utilization.

A number of aromatic compounds produced low growth yields with the slow growing rhizobial strains (Table 3); even after prolonged incubation, the growth yields did not increase in these cases. Quinate and shikimate, in addition to being non-toxic, allowed growth to a full yield. 
In liquid MM, shikimate supported a doubling time of $11 \mathrm{~h}$ for Rhizobium sp. 3241. The same strain had a doubling time of $20 \mathrm{~h}$ with quinate and $6 \mathrm{~h}$ with succinate. Cells grown in the presence of quinate or shikimate were usually small and relatively uniform in appearance, even those of strains like $R$. trifolii strain 2066 which assumed enlarged, pleomorphic shapes in MM supplemented with succinate as sole carbon source.

\section{DISCUSSION}

Auxanographic analysis of diverse rhizobial species revealed considerable metabolic potential overlooked in earlier nutritional surveys of these organisms. Slow growing rhizobia, generally considered to be nutritionally fastidious, were able to use eight to fifteen of the nineteen substrates presented to them as sole sources of carbon and energy; fast growers were able to utilize seven or fewer of the nineteen substrates.

The results suggest that some of the tested compounds may prove useful in elucidating taxonomic relationships among the Rhizobiaceae and in isolating particular groups from the soil. For example, the distinction between fast and slow growers in the ability to utilize adipate warrants further study. Many additional aromatic compounds, existing in free form or covalently bound to other aromatics or sugars, may occur in or around the roots of plants. Growth studies with these compounds may reveal additional nutritional versatility among members of the Rhizobiaceae.

Protocatechuate served as sole source of carbon and energy for the seventeen representatives of the Rhizobiaceae examined. Quinate and shikimate, metabolized via protocatechuate, were commonly used growth substrates. At least six structural genes encode the enzymes that degrade protocatechuate via the 3-oxoadipate pathway (Fig. 2). Conservation of these genes across a broad biological spectrum indicates that there have been selective pressures to maintain them. Possible functions of the genes encoding enzymes of aromatic catabolism are in enabling rhizobia and agrobacteria to survive and to compete effectively in the soil, an environment containing low levels of aromatics derived from the breakdown of lignin and other plant products.

In addition, genes for aromatic catabolism may be involved in bacterial-plant interactions. Most of the aromatic acids used in this survey are widely distributed in plants (Ribereau-Gayon, 1972). Aromatic acids released from plants (Harborne, 1980) may serve as chemo-attractants for rhizobia and agrobacteria. The possibility that aromatic compounds contribute to the metabolism of bacteroids in root nodules is raised by the observation that the total phenolic concentration in alfalfa root nodules exceeds the concentration in roots by more than twofold (Vance, 1978). The hypothesis that aromatics play a role in rhizobial-plant interactions is being tested by obtaining rhizobial strains defective in aromatic catabolism and comparing the properties of wild type and mutant strains.

We are grateful to Drs F. M. Ausubel, J. C. Burton and R. S. Smith of The Nitragen Company, H. H. Keyser of the USDA, and S. Long for kindly providing strains. We also appreciate the help of Dr Gill Lovell in sending seeds of 'Siratro' and vetch.

The Celanese Research Company funded this research, and we are grateful to them for their generous support.

\section{REFERENCES}

Cohen-Bazire, G., Sistrom, W. R. \& Stanier, R. Y. (1957). Kinetic studies of pigment synthesis by nonsulfur purple bacteria. Journal of Cellular and Comparative Physiology 49, 25-68.

ElKan, G. H. (1981). The taxonomy of the Rhizobiaceae. In Biology of the Rhizobiaceae, Supplement 13 of the International Review of Cytology, pp. 1-14. Edited by K. L. Giles \& A. G. Atherly. New York: Academic Press.

EvaNS, W. C. (1947). Oxidation of phenol and benzoic acid by some soil bacteria. Biochemical Journal 41, 373-382.

GlenN, A. R. \& DilworTh, M. J. (1981). Oxidation of substrates by isolated bacteroids and free-living cells of Rhizobium leguminosarum 3841. Journal of General Microbiology 126, 243-247.

Graham, P. H. (1964a). The application of computer techniques to the taxonomy of the root-nodule bacteria of legumes. Journal of General Microbiology 35, $511-517$. 
GrahaM, P. H. (1964b). Studies on the utilisation of carbohydrates and Krebs cycle intermediates by rhizobia, using an agar plate method. Antonie van Leeuwenhoek 30, 68-72.

Graham, P. H. \& Parker, C. A. (1964). Diagnostic features in the characterization of the root-nodule bacteria of legumes. Plant and Soil 20, 383-396.

HARBoRne, J. B. (1980). Plant phenolics. In Secondary Plant Products, Encyclopedia of Plant Physiology, vol. 8, pp. 329-402. Edited by E. A. Bell \& B. V. Charlwood. New York: Springer-Verlag.

Hollis, A. B., Kloos, W. E. \& Elkan, G. H. (1981). DNA:DNA hybridization studies of Rhizobium japonicum and related Rhizobiaceae. Journal of General Microbiology 123, 215-222.

Keane, P. J., KerR, A. \& New, P. B. (1970). Crown gall of stone fruit. II. Identification and nomenclature of Agrobacterium isolates. Australian Journal of Biological Sciences 23, 585-595.

Kersters, K., Deley, J., Sneath, P. H. A. \& SaCkin, M. (1973). Numerical taxonomic analysis of Agrobacterium. Journal of General Microbiology 78, 227239.

KeYSER, H. H., BoHLOOL, B. B., HU, T. S. \& WebER, D. F. (1982). Fast-growing rhizobia isolated from root nodules of soybean. Science 215, 1631-1632.

LEDERBERG, J. (1946). Studies in bacterial genetics. Journal of Bacteriology 52, 503.

Moffett, M. L. \& Colwell, R. R. (1968). Adansonian analysis of the Rhizobiaceae. Journal of General Microbiology 51, 245-266.

Ornston, L. N. \& Parke, D. (1976). Properties of an inducible uptake system for beta-ketoadipate in Pseudomonas putida. Journal of Bacteriology 125, 475-488.
Ornston, L. N. \& Stanier, R. Y. (1966). The conversion of catechol and protocatechuate to betaketoadipate by Pseudomonas putida. I. Biochemistry. Journal of Biological Chemistry 241, 3776-3786.

PARker, C. A., Trinick, M. J. \& Chatel, D. L. (1977). Rhizobia as soil and rhizosphere inhabitants. In $A$ Treatise on Dinitrogen Fixation, Section IV, pp. 311352. Edited by R. W. F. Hardy \& W. S. Silver. New York: Wiley.

Ribereau-Gayon, P. (1972). Plant Phenolics. New York: Hafner.

Riegel, B. \& LiLIENFELD, W. M. (1945). The synthesis of beta-keto esters by decarboxylation of acylated malonic esters. Journal of the American Chemical Society 67, 1273-1275.

Stanier, R. Y., Palleroni, N. J. \& Doudoroff, M. (1966). The aerobic pseudomonads: a taxonomic study. Journal of General Microbiology 43, 159-271.

VANCE, C. P. (1978). Comparative aspects of root and root nodule secondary metabolism in alfalfa. Phytochemistry 17, 1889-1891.

VINCENT, J. M. (1970). A Manual for the Practical Study of Root-nodule Bacteria, p. 75. Oxford: Blackwell Scientific Publications.

VINCENT, J. M. (1977). Rhizobium: general microbiology. In $A$ Treatise on Dinitrogen Fixation, Section $I I I$, pp. 277-366. Edited by R. W. F. Hardy \& W. S. Silver. New York: Wiley.

W ACEK, T. J. \& BRILL, W. J. (1976). Simple, rapid assay for screening nitrogen-fixing ability in soybeans. Crop Science 16, 519-523.

WhITE, L. O. (1972). The taxonomy of the crown-gall organism Agrobacterium tumefaciens and its relationship to rhizobia and other agrobacteria. Journal of General Microbiology 72, 565-574. 